\title{
Reactividad química por intercambio protónico de la serotonina
}

\author{
Christian Cuba1, Walter Solano ${ }^{1}$, Juan Z. Dávalos² \\ ${ }^{1}$ Escuela Profesional de Ciencias Físico Matemáticas, Universidad Nacional de San Cristóbal de Huamanga, \\ Ayacucho, Perú \\ ${ }^{2}$ Instituto de Química-Física "Rocasolano", CSIC, Madrid, España \\ Recibido 14 de junio del 2019, Revisado el 8 de julio de 2019 \\ Aceptado el 13 de julio de 2019
}

DOI: https://doi.org/10.33017/RevECIPeru2019.0004/

\section{Resumen}

Se estudió los procesos de protonación (basicidad, $G B$ ) y desprotonación (acidez, $G A$ ) en fase gaseosa de la serotonina mediante cálculos computacionales utilizando el programa Gaussian D01. El nivel de cálculo usado fue $B 3 L Y P / 6-311++G(d, p)$ y los valores determinados fueron: $G A=1421 \mathrm{kJmol}^{-1}$, para la serotonina desprotonada en el grupo $-\mathrm{NH}-; G B=918,6 \mathrm{kJmol}^{-1}$, para la forma protonada en el grupo etil-amina $\mathrm{NH}_{2}-$ $\mathrm{CH}_{2} \mathrm{CH}_{2}-$.

Descriptores: Acidez/basicidad, serotonina, B3LYP, neurotransmisor.

\section{Abstract}

The serotonin protonation (basicity, $G B$ ) and deprotonation (acidity, $G A$ ) processes in the gas phase were study by computational calculations using Gaussian D01 software. The calculation level used was B3LYP/6$311++G(d, p)$ and the determined values were: $G A=1421 \mathrm{kJmol}^{-1}$, for the deprotonated serotonin in the $-\mathrm{NH}-$ group; $G B=918,6 \mathrm{kJmol}^{-1}$, for the protonated in the ethyl-amine group $\mathrm{NH}_{2}-\mathrm{CH}_{2} \mathrm{CH}_{2}-$.

Keywords: Acidity/basicity, serotonine, B3LYP, neurotransmitter.

\section{Introducción}

En el Sistema Nervioso Central (SNC), la serotonina (Fig.1) tiene una función neurotransmisora con diferentes subtipos de receptores 5hidroxitriptamina (5-HT). Este neurotransmisor influye en numerosos procesos fisiológicos como el sueño, cognición, actividad motora, regulación de la temperatura corporal, nocicepción, apetito, comportamiento sexual, etc. En el sistema cardiovascular, gastrointestinal y de agregación plaquetaria, la serotonina juega un papel regulador de la función del músculo liso.

La serotonina puede presentar varias formas "cargadas": neutras, catiónicas (protonadas), aniónicas (desprotonadas) y zwiteriónicas [1]. Nagy y col. [2] encontraron que la serotonina en disolución acuosa a $\mathrm{pH}=7,4$ está presente principalmente en su forma protonada $(99,7 \%)$ y para $\mathrm{pH}>9$ prevalecen las formas neutras [3]. El sitio preferencial de protonación sería la cadena etilamina [4]. Zweir y col [5] han encontrado que las estructuras tipo "Gauge" del $\mathrm{NH}_{2}-$ sobre el pirrol son las más estables.

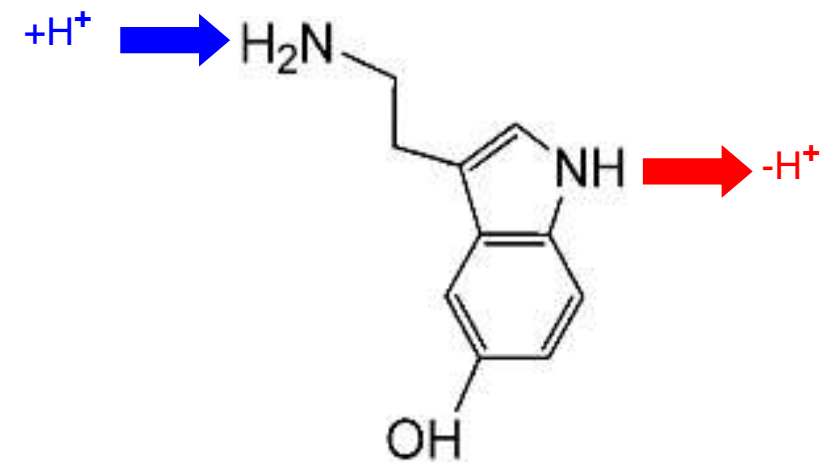


Figura 1: Estructura de la serotonina, mostrando sitios preferenciales de protonación $y$ desprotonación.

Es importante mencionar que las reacciones por transferencia protónica juegan un papel importante en una amplia variedad de procesos, biológicos, industriales y medio ambientales [6] y son cuantificados por magnitudes termodinámicas como la acidez y la basicidad/afinidad protónica.

La acidez en fase gas (GA) de un ácido prótico $A H$ y la basicidad (GB) de una base $B$, vienen definidas como el cambio en la energía libre de Gibbs para las reacciones (1) y (2) respectivamente,

$$
\begin{gathered}
A H(g) \rightarrow A^{-}(g)+H^{+}(g) \\
\Delta_{a c i d} G^{0}=G A, \Delta_{a c i d} H^{0}, \Delta_{a c i d} S^{0} \\
B H^{+}(g) \rightarrow B(g)+H^{+}(g) \\
\Delta_{p} G^{0}=G B, P A=\Delta_{p} H^{0}, \Delta_{p} S^{0}
\end{gathered}
$$

El correspondiente cambio en la entalpía y entropía en la reacción (1) se denominan $\left(\Delta_{\text {acid }} H^{0}\right)$ y $\left(\Delta_{\text {acid }} S^{0}\right)$, respectivamente; mientras para la reacción (2) se denominan afinidad protónica $(P A)$ y entropía de protonación, $\left(\Delta_{p} S^{0}\right)$.

Los valores reportados, particularmente los de la acidez en fase gas, son escasos o no están descritos en la bibliografía.

\section{Metodología}

Se utilizó el programa Gaussian D01 implementado en los Clusters TRUENO e HIDRA del CSIC. Utilizamos el funcional B3LYP con una base 6$311++G(d, p)$ para el análisis conformacional, optimizando la geometría de las especies neutras e iónicas y determinando sus correspondientes funciones de estabilidad termodinámica, entalpía y energía libre de Gibbs ( $H$ y $G$ ), respectivamente.

\section{Resultados}

En la figura 2 se muestra la estructura y distribución de cargas del rotámero neutro más estable y abundante $(\sim 11 \%)$ de la serotonina, similar a la estructura determinada por Zweir y col [5] notándose dos sitios activos de desprotonación (-
$\mathrm{NH}-\mathrm{y}-\mathrm{OH}$ ) y dos sitios activos de protonación ($\mathrm{NH}-$ y $\mathrm{NH}_{2}-$ ).

La serotonina neutra se desprotona preferentemente en el grupo -NH- (Fig.3) y el valor de su $G A$ es de $1421 \mathrm{kJmol}^{-1}$ (Tabla 1). Esta localización es $16,2 \mathrm{kJmol}^{-1}$ más ácida que en el grupo $-\mathrm{OH}$. La serotonina es 47 y $19 \mathrm{kJmol}^{-1}$ más ácida que el pirrol e indol, respectivamente (cuyas GAs fueron tomados del NIST [7]).

La serotonina neutra se protona preferentemente en el grupo etil-amina $\mathrm{NH}_{2}-\mathrm{CH}_{2} \mathrm{CH}_{2}-$ (Fig. 4) y el valor de su basicidad es $G B=918,6 \mathrm{kJmol}^{-1}$. Esta localización es $81,2 \mathrm{kJmol}^{-1}$ más básica que en el grupo $-\mathrm{NH}$-. Por otra parte, la serotonina es $40,6 \mathrm{kJmol}^{-1}$ más básica que la etilamina [7]. Es de mencionar que el valor que encontramos para la afinidad protónica $P A$ es $46,1 \mathrm{kJmol}^{-1}$ más bajo que el obtenido por Omidyan y col. [4] al nivel MP2/ $\mathrm{cm}^{3}$ pVDZ.

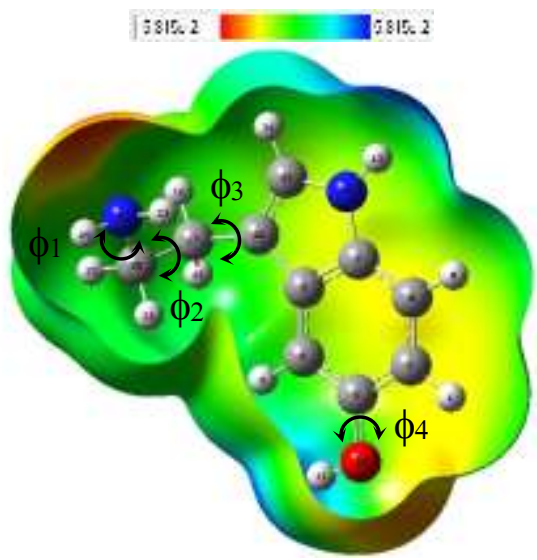

Figura 2: Estructura y distribución de cargas del rotámero más estable de la serotonina neutra en fase gas. Optimizada a nivel B3LYP/6-311++G(d,p), $\phi_{1}=174.4^{\circ}, \phi_{2}=64.5^{\circ}, \phi_{3}=-102.2^{\circ}, \phi_{4}=-179.9^{\circ}$.

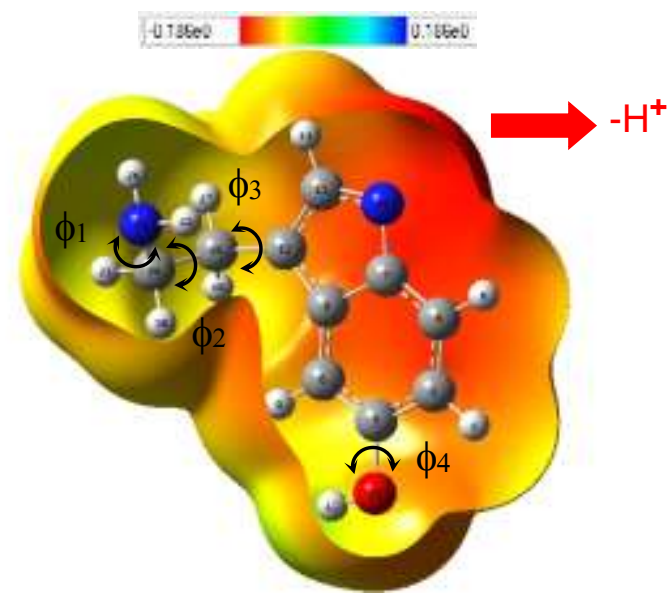


Figura 3: Distribución de cargas y estructura del rotámero más estable de la serotonina desprotonada, en fase gas, optimizada a nivel $B 3 L Y P / 6-311++G(d, p)$,

$\phi_{1}=59^{\circ}, \phi_{2}=59.2^{\circ}, \phi_{3}=-102.1^{\circ}, \phi_{4}=-178.4^{\circ}$.

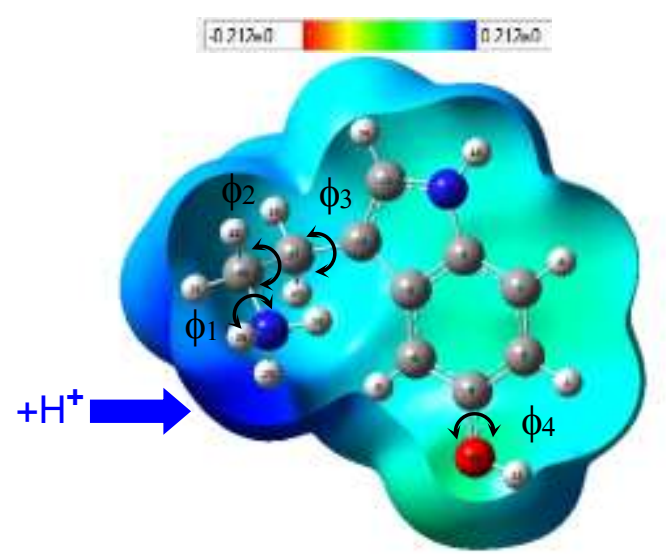

Figura 4: Rotámero más estable de la serotonina protonada en fase gas, optimizada a nivel B3LYP/6$311++G(d, p), \quad \phi_{1}=44.7^{\circ}, \phi_{2}=-53.2^{\circ}, \phi_{3}=-92.9^{\circ}$, $\phi_{4}=-5.4^{\circ}$

Tabla 1: Acidez y Basicidad obtenidas al nivel $B 3 L Y P / 6-311++G(d, p)$ de la serotonina.

\begin{tabular}{|l|r|r|}
\hline \multicolumn{3}{|c|}{ Cálculo B3LYP/6-311++G(d,p) } \\
\hline \multirow{3}{*}{ Desprotonación } & $\Delta_{\text {acid }} G^{0}(G A) / \mathrm{kJ} \mathrm{mol}^{-}$ & \\
& & 1421 \\
\cline { 2 - 3 } & $\Delta_{\text {acid }} H^{0} / \mathrm{kJmol}^{-1}$ & 1449,9 \\
\cline { 2 - 3 } & $\Delta_{\text {acid }} S^{0} / \mathrm{Jmol}^{-1}$ & 96.9 \\
\hline \multirow{3}{*}{ Protonación } & $\Delta_{\mathrm{p}} G^{0}(G B) / \mathrm{kJmol}^{-1}$ & 918,6 \\
\cline { 2 - 3 } & $\Delta_{\mathrm{p}} H^{0}(P A) / \mathrm{kJmol}^{-1}$ & 957,7 \\
\cline { 2 - 3 } & $\Delta_{\mathrm{p}} S^{0} / \mathrm{Jmol}^{-1}$ & 130,9 \\
\hline
\end{tabular}

\section{Conclusiones}

La serotonina, al igual que el pirrol y el indol, se desprotonan preferentemente en el grupo $-\mathrm{NH}-$; pero es $19 \mathrm{kJmol}^{-1}$ más ácida que el indol y ésta a su vez es $28 \mathrm{kJmol}^{-1}$ más ácida que el pirrol.

Por otra parte, la serotonina se protona preferentemente en el nitrógeno del grupo aminoetilo y es $40,6 \mathrm{kJmol}^{-1}$ más básica que la etilamina.

\section{Referencias}

[1] P. Manivet and col, Chem. Phys. Lett. 420 (2006) 538-544.

[2] P.I. Nagy, G. Alagona, C. Ghio and K.Takacs-Novak, J. Am. Chem. Soc.125 (2003) 2770-2785.
[3] P.I. Nagy, Int. J. Mol. Sci. 15 (2014) 1956219633.

[4] R. Omidyan, Z. Amanollahi and G. Azimi, Spectrochim Acta A Mol BiomolSpectrosc. 182 (2017) 8-16.

[5] T.A. LeGreve, E.E. Baquero and T.S. Zwier, J. Am. Chem. Soc. 129 (2007) 4028-4038.

[6] J.F.J. Todd, Int. J. Mass Spectrom. Ion Process.142 (1995) 209-240.

[7] NIST Chemistry Webbook. Standard Reference Database Number 69; P.J.Linstrom, W.G.Mallard, Release June 2005: National Institute of Standards and Technology, GaithersburgMD, 20899 (https://webbook.nist.gov/).

E-mail: $\quad$ chris cubklium@hotmail.com, walmasolrey@gmail.com, jdavalos@iqfr.csic.es 